\title{
The Importance of the Left Occipitotemporal Cortex in Developmental Dyslexia
}

\author{
Lisa Kronbichler ${ }^{1,2} \cdot$ Martin Kronbichler ${ }^{1,2}$ \\ Published online: 19 January 2018 \\ (C) The Author(s) 2018. This article is an open access publication
}

\begin{abstract}
Purpose of Review Developmental dyslexia is characterized by an impaired acquisition of fluent and skilled reading ability. Numerous studies have explored the neural correlates of this neurodevelopmental disorder, with most classic accounts strongly focussing on left temporoparietal regions. We will review recent findings from structural and functional MRI studies that suggest a more important role of occipitotemporal cortex abnormalities in dyslexia.

Recent Findings Recent findings highlight the role of the occipitotemporal cortex which exhibits functional as well as structural abnormalities in dyslexic readers and in children at risk for dyslexia and suggest a more central role for the occipitotemporal cortex in the pathophysiology of dyslexia.

Summary We demonstrate the importance of the occipitotemporal cortex in for understanding impaired reading acquisition and point out how future research might enhance our understanding of functional and structural impairments in the reading network via large-scale data analysis approaches.
\end{abstract}

Keywords Developmental dyslexia $\cdot$ Occipitotemporal cortex $\cdot$ Visual word form area $\cdot$ Brain $\cdot$ MRI $\cdot$ fMRI

\section{Introduction}

Developmental dyslexia (henceforth, dyslexia) designates impaired acquisition of reading skills which is not merely accounted for by mental age, sight defects, or insufficient schooling [1]. Affected individuals show difficulties in reading comprehension, word decoding, and recognition and similar tasks that require adequate reading skills [2, 3]. Dyslexia, which describes the difficulty in decoding the spelling and pronunciation of words, must be distinguished from reading comprehension impairments, where readers have problems understanding the meaning of what they read. Family studies show that dyslexia is heritable and has a strong genetic component [4]. Dyslexia lacks a concrete etiology and is determined via dimensional classification schemes: it describes the

This article is part of the Topical Collection on Learning Disorders

Martin Kronbichler

martin.kronbichler@sbg.ac.at

1 Centre for Cognitive Neuroscience and Department of Psychology, University of Salzburg, Hellbrunnerstraße 34, Salzburg 5020, Austria

2 Neuroscience Institute, Christian Doppler Medical Centre, Paracelsus Medical University, Ignaz-Harrer Straße 79, Salzburg 5020, Austria lower end of a continuous distribution that ranges from poor outcome to optimal outcome in word reading ability. However, in clinical practice, a rather arbitrary cutoff point is normally set to separate dyslexia from subclinical reading deficits. Reading skills are commonly related to general intelligence, although the importance of the discrepancy between reading ability and general intelligence has been refuted by recent research $[5,6]$.

Besides several auditory, visual, and motor dysfunction hypotheses, the prevalent (and most consistent [7]) cognitive explanatory approach for dyslexia is the phonological deficit hypothesis. It postulates a specific deficit to represent, access, and process speech sounds caused by inherent dysfunctions of cortical areas specialized in phonology and reading. Word reading difficulties differ somewhat across languages since their orthographies vary in the transparency of their grapheme-phoneme mappings. It is therefore easier to read Finnish or German words (transparent orthographies) as compared to English words (opaque orthography) [8], and this affects dyslexic as well as normal readers $[9,10]$. Several attempts have been made to identify subtypes of dyslexia [1, $11 \bullet \bullet$. Critically, none of these typologies is universally accepted in the field of dyslexia research. Dyslexia is comorbid with other neurodevelopmental disorders like ADHD and 
dyscalculia and therefore it is not surprising that there is a solid correlation between reading and mathematical abilities and that these disorders might share common patterns of brain alterations [12].

In most classic accounts of brain abnormalities in dyslexia, the importance of the left temporoparietal cortex is especially highlighted, since this brain region has been closely linked with the phonological processing deficits in dyslexia $[13,14,15 \cdot]$. Although left ventral posterior occipitotemporal dysfunctions are regularly discussed, those were often seen as secondary brain deficits in dyslexia, as it was assumed that phonological processing deficits reflected in temporoparietal dysfunctions would cause disrupted development of the left occipitotemporal cortex (OTC). This account was also based on the idea that the temporoparietal cortex is especially important in the early stage of reading acquisition with phonologically based word decoding and that the OTC only emerges in later reading development. In this paper, we will review recent evidence that points to a more central role of the left OTC in dyslexia which suggests that functional and structural abnormalities of this region may constitute the most reliable and important neural correlate of developmental dyslexia. We will primarily review functional and structural MRI studies and mostly studies with alphabetic orthographies, as there are still (in our opinion) not enough studies to evaluate the consistency of neural abnormalities in dyslexia in other orthographies. One should note, however, that there are some reports showing that Chinese dyslexic readers do not show abnormalities in posterior brain regions, but rather in the middle frontal gyrus [16], although the left OTC is reliably involved in skilled reading also in logographic scripts [17]. Another study found comparable left OTC dysfunction in Chinese and English dyslexic readers [18].

\section{Brain Activation Abnormalities}

The occipitotemporal (including the visual word form area; VWFA), temporoparietal, and left frontal regions regularly emerge in fMRI studies on dyslexia and are identified as important core regions for reading [1, 15•, 19-22]. Temporoparietal cortex (TPC) activation is often found in studies (and meta-analyses), although with some limitations. First, TPC activation seems to vary with reading proficiency in impaired and normal reading: Richlan et al. [23] identified underactivation in the left temporal areas in adult dyslexic readers whereas this effect was not replicated for children studies. Conversely, convergent temporal activation was shown for healthy infant but not for adult readers [24•]. Second, it is not yet clear how TPC abnormalities vary with orthographic depth: Although TPC underactivation in dyslexic readers was identified in deep and shallow orthographies in a recent meta-analysis [25•*], this effect heavily relied on tasknegative activation in dyslexic participants and group differences disappeared when deactivation relative to baseline was excluded from the analyses. Such inconsistencies appear incompatible with the prominent role the TPC is assigned to in neural models of dyslexia.

By contrast, the OCT shows convergent activation irrespective of reading proficiency as demonstrated in robust activation in child as well as adult readers, which highlights the role of the OCT not only during proficient reading but also during reading acquisition (meta-analysis [24•]). In a similar vein, child as well as adult dyslexic readers reveal convergent underactivation in the left ventral OTC (meta-analysis [23]). Abnormal activation in dyslexic readers in OTC might be unaffected by orthographic depth, since decreased neural response was identified in deep (English) as well as in shallow (e.g., Dutch, Italian) orthographies (metaanalysis [25•0]). Besides consistent results on less activation in the left OTC in dyslexic readers, dyslexia is also associated with an abnormal neural response profile: Neural activation in dyslexic children is assumed to differentiate to a lesser extent between letters and other visual stimuli and between different types of words: Neural activation in OTC during the visual presentation of words as compared to false fonts is higher in children with normal reading abilities than in dyslexic children. They also showed a decreased differentiation between orthographically familiar and unfamiliar forms of real words [26]. An abnormal response profile of the OTC in dyslexia during visual word processing in terms of a decreased specialization was also found in subsequent fMRI studies, and can be considered as one of the most robust findings [27-29].

Support for the important role of the left OTC in dyslexia is also provided by training studies on children with reading disabilities, showing that systematic reading interventions (50 min per day) increased not only reading fluency but also neural response in the occipitotemporal cortex [30]. This effect was not replicated for "communal interventions" (i.e., interventions often provided within school settings). Another study on dyslexic readers investigated the effects of training in the domains of phonology, attention, or visual word recognition on neural response [31•]. The authors found that, irrespective of training type, significant increase in activation was only found in occipitotemporal regions, which (once more) highlights the importance of the OTC in literacy skills. ERP assessments in prereaders could show that occipitotemporal sites exhibit a delayed N1 component in infants who develop dyslexia later on in development [32]. In a similar vein, print knowledge was identified as a reliable predictor of later reading performance as early as reception class level at the ages of $\sim 4$ and 6 [33]. At the neuronal level, fMRI assessments during orthographic processing (letter vs. false font) in prereaders revealed a decreased specialization for letters in posterior dorsal regions [34]. Similar to findings of the training study of Shaywitz et al. [30] mentioned above, abnormal neural activation was improvable by supplementary reading intervention. Additional support for abnormal posterior brain activation prior to reading onset was provided by Raschle et al. [35]: 
They examined neural activation in kindergarten children during a sound matching task and found reduced activation in the OTC and TPC in infants with a family history of dyslexia.

Taken together, developmental dyslexia is frequently associated with a decreased specialization for letters and words in the OTC. Decreased neural response during reading-related processes in dyslexia seems to be invariant to orthographic depth and is robustly identified in adult as well as infant reading. Altered activation is also found in prereading studies suggesting early developmental brain alterations prior to reading acquisition [36•]. Notably, although reading abilities are often defined in relation to general intelligence, neuroimaging studies revealed evidence for the contrary: Tanaka et al. [37] assessed phonological processing in poor readers with either high or low IQ and found that both groups exhibited similar patterns of reduced neural activation in the OTC and TPC. With respect to the relationship between reading comprehension and word reading, one fMRI study provided evidence that decreased activation of the left OTC during reading is specifically associated with problems in word reading and not with impaired reading comprehension [38]. In a very recent study, Perrachione et al. [39••] found diminished neural adaptation in dyslexic readers compared to that in normal readers while processing written words in the OTC. Neural adaptation is assumed to reflect an efficient processing of sensory input and it is defined as the decreased blood oxygen level dependent (BOLD) response towards a repeatedly presented stimulus in regions critically involved in the processing of the investigated stimulus category. Strikingly, diminished neural adaptation was not restricted to reading-specific material but was also identified during visual object and face perception. Assessment of functional MRI during visual processing found further support for the notion that deficient activation in dyslexia may not be restricted to reading material but rather reflects a more general impairment of the OTC: Dyslexic readers exhibited decreased OTC activation not only for word-like stimuli but also for numbers and abstract symbol strings [40•]. Decreased activation for non-word stimuli was also found in an earlier study that reported diminished activation not only in the left occipitotemporal word-selective regions for visual words but also in the right fusiform face area in response to visual faces in dyslexic children [41]. Collectively, these recent findings suggest that abnormal left OTC function in dyslexia is not restricted to visual word processing and may therefore not be solely caused by a lack of reading experience in dyslexic readers, as also suggested by other fMRI findings reviewed in this section.

\section{Structural Brain Abnormalities}

First neuroanatomical assessments of structural brain abnormalities in dyslexia arose from post-mortem investigations
[42]. Abnormalities included a reduced leftward asymmetry of the planum temporale and ectopias located in the perisylvian regions. With the beginning of neuroimaging in living individuals, voxel-based morphometry (VBM) assessments showed gray matter abnormalities in the ventral OTC $[43,44]$, although the direction of altered findings was rather inconsistent. Furthermore, such gray matter abnormalities could not be replicated thoroughly [45]. On the contrary, gray matter reduction in dyslexic readers in OTC was identified in a meta-analysis by Linkersdörfer et al. [46]: The authors conducted a meta-analysis on VBM studies which revealed that abnormal neural activation and gray matter alterations in dyslexia overlapped in the left fusiform gyrus. In a similar fashion, Altarelli et al. [47] examined cortical thickness of dyslexic children around their individual peak of functional activation towards visually presented words. Dyslexic children showed decreased cortical thickness in word-selective OTC but not in other cortical areas. Notably, this effect was mainly driven by differences in healthy vs. impaired female readers. Decreased cortical thickness (and increased gyrification) in the OTC is also shown by a very recent study of Williams et al. [48]. There is also evidence that gray matter abnormalities in the OTC are specific for dyslexia and not found in readers with a specific reading comprehension impairment but no deficit in word reading per se [49].

Similar to functional MRI investigations, structural assessments show variations in the neuroanatomy of reading-related regions in at-risk prereaders. Structural alterations prior to reading acquisition include atypically small sulcal basins [50•] and reduced gray matter volume in the OTC as well as TPC [51, 52]. Here, gray matter alterations were associated with a delay in language acquisition [52] and a family history of developmental dyslexia [51, 52]. Volume indices also correlated positively with rapid automatized naming tasks in these cortical areas [51].

Genetic imaging studies have also demonstrated an association between OTC structure and genetic markers of dyslexia [4]. Skeide et al. [53•] investigated single-nucleotide polymorphisms (variations in certain DNA sequences) often associated with literacy performance in 19 genes in kindergarten schoolchildren (prereaders; 5-6 years). The NRSN1 gene was related to gray matter volume in the VWFA. The genetically associated volume profile of the VWFA (gVWFA) was able to classify readers into dyslexic and control individuals. Furthermore, gVWFA accurately distinguished between later dyslexic and normal reading skills in the prereader sample. In another recent genetic imaging study, a link was found between a genetic risk factor for dyslexia and dyscalculia and decreased volume and brain activation of the fusiform gyrus in the left OTC [54].

Despite the large number of studies pointing to the importance of structural abnormalities in the OTC for dyslexia, it should be noted that the literature on structural brain abnormalities in dyslexia is plagued by inconsistencies and failures to replicate (for an excellent recent critical review, see [11••]). 
These inconsistencies are also reflected in a large-scale study of structural abnormalities based on a multi-site dyslexia dataset collected via the Dyslexia Data Consortium. This analysis showed that most differences in gray and white matter volume disappeared when the analysis adjusted for differences in whole brain volume and that dyslexia was associated with more heterogeneity of gray matter volume in most regions of the brain $[55 \cdot \bullet]$.

\section{Structural Brain Connectivity}

Dyslexia was early conceptualized as a disconnection syndrome [56], consistent with the explanation that impaired reading might be closely associated with impaired connectivity and disturbed white matter tracts between reading-related brain areas. Accordingly, numerous studies have explored structural connectivity in dyslexia using diffusion-weighted MRI [57]. As in studies exploring functional MRI and structural MRI, early assessments mainly focused on connectivity involving the left TPC, including the arcuate fasciculus and the corona radiata, because of their assumed link with phonological processing. A number of findings from these studies indicated abnormalities in white matter tracts but with considerable inconsistency on which track is specifically involved $[11 \bullet \cdot$. Regarding the OTC, recent studies provided some evidence for white matter abnormalities. For example, familial risk for dyslexia in prereading children was mainly associated with deficits in a left ventral white matter tract, the left inferior fronto-occipital fasciculus [58 ${ }^{\bullet}$, and integrity of this ventral tract was related to both familial risk for dyslexia and later reading ability in a longitudinal DWI study [59]. Interestingly, integrity of this ventral pathway was found to be associated with performance on an orthographic processing task [60]. However, there are also inconsistent results from studies indicating no association between integrity of ventral white matter tracts and risk for dyslexia in prereaders, but rather highlight an association between dorsal tracts and dyslexia risk [61]. In summary, there is inconsistent evidence for abnormalities of ventral white matter tracts in the OTC in dyslexia and reading impairment. Generally, although there is a consensus on the importance of white matter tract abnormalities in dyslexia, there is still no clear consensus on which specific white matter abnormalities are reliably associated with dyslexia. It should be noted that DWI studies can differ in numerous aspects [11••], which makes formal meta-analysis across studies difficult and may partly explain the lack of a clear consensus.

\section{Functional Brain Connectivity}

To date, relatively few fMRI studies have reported findings on impaired functional brain connectivity in dyslexia, despite the clear interest on disordered communication between brain regions. With respect to the left OTC, we have recently shown that this region reveals less connectivity with the left inferior frontal regions [62・•]. Importantly, this reduction of functional connectivity of the OTC was not merely found during two different reading tasks but also during the task-free resting state, thus highlighting a general and permanent disruption of the left OTC in dyslexia. Comparable results were reported in other fMRI connectivity studies during reading tasks [29, $63,64]$. One recent resting fMRI connectivity study with Chinese dyslexic children also reported decreased connectivity of the left OTC with the left frontal regions [65]. Another study reported widespread functional connectivity abnormalities in dyslexia using whole-brain data-driven analysis approach. They also identified reduced coupling between the ventral visual regions in the OTC and reduced coupling between the visual and prefrontal regions [66]. However, some studies did not identify impaired functional connectivity involving the left OTC but revealed other abnormalities in dyslexia during reading or rest [67-70]. Compared to other brain disorders, there are relatively few fMRI connectivity and especially resting state connectivity studies on dyslexia. Even fewer studies employ effective connectivity techniques like Dynamic Causal Modelling (DCM), which would allow for a more targeted analysis of connectivity abnormalities and the role of top-down and bottom-up connectivity in dyslexia and which turned out promising in understanding connectivity during visual word processing [71]. The small number of DCM studies in dyslexia does not yet show a consistent pattern for the OTC (or other regions of the reading network) [72, 73•]. Clearly, more and larger studies are needed to characterize potential abnormalities in connectivity of the OTC in dyslexia more reliably, also because studies in non-impaired readers suggest that the role of the OTC and its emergence during development is strongly determined by structural and functional connectivity $[74,75]$.

\section{Functions of the OTC in Non-Impaired Reading}

The OTC is consistently identified in studies assessing literacy skills in normal and dyslexic reading. This area is assumed to accommodate a functional region - VWFA - which computes representations of visually presented words irrespective of variances in size, font, location, and case [76]. Initial accounts of the VWFA assumed that it is mainly responsible for prelexical orthographic processing and does not respond to auditory words [77]. However, recent neuroimaging findings account this of the VWFA: For example, a number of findings now strongly argue for orthographic (whole word) representations in the sense of an orthographic lexicon within the VWFA [78, 79]. Furthermore, VWFA seems to be involved in accessing the orthography of auditorily presented words 
[80]. Assuming that abnormal VWFA activation is also related to disturbed auditory processes, this might be an interesting link to spelling deficits identified in some dyslexics or the finding that lesions in the VWFA are often accompanied by acquired alexia.

Variable response properties suggest that VWFA activation also contributes to other functions besides the mere visual representation of words. In their "The interactive account of the ventral occipitotemporal contributions to reading," Price and Devlin [81] propose that the function of this region varies depending on its interaction with other areas. Therefore, top-down input from auditory processes or lexical information might alter VWFA activation for efficient stimulus processing. An alternative approach is that there exist two functionally different regions within the OTC $[82,83]$ : One that is relevant for the graphemic description of a word, irrespective of its location, font, or size. This region corresponds to what is often termed VWFA [82]. The other region is located lateral to the left of the VWFA and is engaged in a variety of lexical tasks and responds to written and spoken words $[82,83]$. It is therefore assumed to be modality independent and to link semantics to lexical representation for written or oral output. Although there is some support for this approach, this functional distinction needs future investigations since - due to their spatial proximity - these two regions are confounded in previous studies and task-dependent individual localizations of the VWFA complicate a retrospective separation even more.

\section{Conclusions}

In summary, the OTC is most consistently identified in infant and adult readers irrespective of orthographic depth and is even shown in fMRI assessments of non-alphabetic writing systems. Dyslexic readers not merely show decreased neural response in the OTC but rather indicate unspecific activation towards a reading material. Although less consistent, several structural MRI assessments highlight the role of structural alterations in the OTC in dyslexic readers. Strikingly, structural as well as functional alterations become evident even before reading acquisition in preschool children and are able to accurately predict later reading disabilities. The central importance of the OTC in reading and reading-related skills like spelling and (rapid) object naming might be one potential explanation for the relatively broad literacy impairments of dyslexia. Connectivity impairments between the OTC and frontal areas might be indicative of a disrupted linkage between orthographic and phonological word representations what in turn leads to inefficient and slow reading performance.

Considering the evidence of abnormalities in dyslexia in various research domains ranging from behavioral RT measures to functional brain activation and even genetic factors, it seems unlikely that there is a single mechanistic explanation for dyslexia. Additionally, there is an ongoing discussion about whether there are qualitative differences in the neuronal mechanisms that underlie dyslexia, meaning that dyslexic symptoms might be based upon slightly diverging "neuronal subtypes" of dyslexia. The assumption of different neuronal subtypes might account for the sometimes inconsistent findings produced in this field. To illustrate, it remains up to future studies to show how occipitotemporal abnormalities relate to temporoparietal and frontal activation patterns and why certain tasks elicit decreased neural response in dyslexic readers whereas others do not. Furthermore, alterations in functional activation and structure might also be influenced by comorbid disorders like ADHD and dyscalculia and subclinical traits; at the moment, there is not much brain data directly exploring the relationship between dyslexia and other neurodevelopmental disorders and between reading ability and other skills and traits.

Advanced approaches might provide suitable methods to classify dyslexic subgroups in a data-driven manner based on brain function, connectivity, and structure: Such methods are already successfully applied in other psychiatric disorders [84]. Besides, advanced developments in the field of computational neuroanatomy and quantitative MRI might also be of great relevance for an improved and more sensitive analysis of brain structure [85]. In general, there is an urgent need for a careful integration of large-scale functional and structural MRI datasets on dyslexia and increased data sharing. The first steps and promising results have already been provided by structural MRI data collected by the Dyslexia Data Consortium [55••]. But, large-scale, publicly available datasets are also needed for functional MRI data; such data sharing already had a large impact on research on the brain basis of another neurodevelopmental disorder, for example, in the field of autism research [86].

Acknowledgements Open access funding provided by Paris Lodron University of Salzburg.

\section{Compliance with Ethical Standards}

Conflict of Interest The authors declare that they have no competing interests.

Human and Animal Rights and Informed Consent This article does not contain any studies with human or animal subjects performed by any of the authors.

\section{References}

Papers of particular interest, published recently, have been highlighted as:

- Of importance

•. Of major importance

1. Peterson RL, Pennington BF. Developmental dyslexia. Annu Rev Clin Psychol. 2015;11(1):283-307. https://doi.org/10.1146/ annurev-clinpsy-032814-112842. 
2. Ellis AW. Reading, writing and dyslexia (classic edition): a cognitive analysis. Psychology Press; 2016.

3. Aaron PG. Dyslexia and hyperlexia: diagnosis and management of developmental reading disabilities. Springer Science \& Business Media; 2012.

4. Mascheretti S, De Luca A, Trezzi V, Peruzzo D, Nordio A, Marino $\mathrm{C}$, et al. Neurogenetics of developmental dyslexia: from genes to behavior through brain neuroimaging and cognitive and sensorial mechanisms. Transl Psychiatry. 2017;7(1):e987. https://doi.org/10. 1038/tp.2016.240.

5. Tunmer W, Greaney K. Defining dyslexia. J Learn Disabil. 2010;43(3):229-43. https://doi.org/10.1177/0022219409345009.

6. O'Malley KJ, Francis DJ, Foorman BR, Fletcher JM, Swank PR. Growth in precursor and reading-related skills: do low-achieving and IQ-discrepant readers develop differently? Learn Disabil Res Pract. 2002;17(1):19-34. https://doi.org/10.1111/1540-5826. 00029.

7. Ramus F, Rosen S, Dakin SC, Day BL, Castellote JM, White S, et al. Theories of developmental dyslexia: insights from a multiple case study of dyslexic adults. Brain. 2003;126(4):841-65. https:// doi.org/10.1093/brain/awg076.

8. Ziegler JC, Bertrand D, Tóth D, Csépe V, Reis A, Faísca L, et al. Orthographic depth and its impact on universal predictors of reading: a cross-language investigation. Psychol Sci. 2010;21(4):551-9. https://doi.org/10.1177/0956797610363406.

9. Landerl K, Wimmer H, Frith U. The impact of orthographic consistency on dyslexia: a German-English comparison. Cognition. 1997;63(3):315-34. https://doi.org/10.1016/S0010-0277(97) 00005-X.

10. Aro M, Wimmer H. Learning to read: English in comparison to six more regular orthographies. Appl Psycholinguist. 2003;24:621-35.

11.• Ramus F, Altarelli I, Jednoróg K, Zhao J, di Covella LS. Neuroanatomy of developmental dyslexia: pitfalls and promise. Neurosci Biobehav Rev. 2017. An extremely detailed and critical review of recent problems in structural MRI studies on dyslexic brain abnormalities and inconsistency in results between studies.

12. Ashkenazi S, Black JM, Abrams DA, Hoeft F, Menon V. Neurobiological underpinnings of math and reading learning disabilities. J Learn Disabil. 2013;46(6):549-69. https://doi.org/10. 1177/0022219413483174.

13. Pugh KR, Mencl WE, Jenner AR, Katz L, Frost SJ, Lee JR, et al. Functional neuroimaging studies of reading and reading disability (developmental dyslexia). Ment Retard Dev Disabil Res Rev. 2000;6(3):207-13. https://doi.org/10.1002/1098-2779(2000)6: $3<207::$ AID-MRDD8>3.0.CO;2-P.

14. Démonet J-F, Taylor MJ, Chaix Y. Developmental dyslexia. Lancet. 2004;363(9419):1451-60. https://doi.org/10.1016/S0140-6736(04) 16106-0.

15. Xia Z, Hancock R, Hoeft F. Neurobiological bases of reading disorder part I: etiological investigations. Lang Linguist Compass. 2017;11(4):e12239. Informative review on neuroimaging approaches to addressing the causality of neural signature in dyslexia. https://doi.org/10.1111/lnc3.12239.

16. Siok WT, Niu Z, Jin Z, Perfetti CA, Tan LH. A structural-functional basis for dyslexia in the cortex of Chinese readers. Proc Natl Acad Sci U S A. 2008;105(14):5561-6. https://doi.org/10.1073/pnas. 0801750105.

17. Rueckl JG, Paz-Alonso PM, Molfese PJ, Kuo W-J, Bick A, Frost SJ, et al. Universal brain signature of proficient reading: evidence from four contrasting languages. Proc Natl Acad Sci U S A. 2015;112(50):15510-5. https://doi.org/10.1073/pnas.1509321112.

18. Hu W, Lee HL, Zhang Q, Liu T, Geng LB, Seghier ML, et al. Developmental dyslexia in Chinese and English populations: dissociating the effect of dyslexia from language differences. Brain. 2010;133(6):1694-706. https://doi.org/10.1093/brain/awq106.
19. Norton ES, Beach SD, Gabrieli JDE. Neurobiology of dyslexia. Curr Opin Neurobiol. 2015;30:73-8. https://doi.org/10.1016/j. conb.2014.09.007.

20. Maisog JM, Einbinder ER, Flowers DL, Turkeltaub PE, Eden GF. A meta-analysis of functional neuroimaging studies of dyslexia. Ann N Y Acad Sci. 2008;1145(1):237-59. https://doi.org/10.1196/ annals.1416.024.

21. Richlan F, Kronbichler M, Wimmer H. Functional abnormalities in the dyslexic brain: a quantitative meta-analysis of neuroimaging studies. Hum Brain Mapp. 2009;30(10):3299-308. https://doi.org/ 10.1002/hbm. 20752.

22. Paulesu E, Danelli L, Berlingeri M. Reading the dyslexic brain: multiple dysfunctional routes revealed by a new meta-analysis of PET and fMRI activation studies. Front Hum Neurosci. 2014;8:830.

23. Richlan F, Kronbichler M, Wimmer H. Meta-analyzing brain dysfunctions in dyslexic children and adults. NeuroImage. 2011;56(3): 1735-42. https://doi.org/10.1016/j.neuroimage.2011.02.040.

24. Martin A, Schurz M, Kronbichler M, Richlan F. Reading in the brain of children and adults: a meta-analysis of 40 functional magnetic resonance imaging studies. Hum Brain Mapp. 2015;36(5): 1963-81. Meta-analytic evidence for the important role of OTC during reading acquisition and proficient reading. https://oi.org/10.1002/hbm.22749.

25.• Martin A, Kronbichler M, Richlan F. Dyslexic brain activation abnormalities in deep and shallow orthographies: a meta-analysis of 28 functional neuroimaging studies. Hum. Brain Mapp. 2016;37(7):2676-99. Meta analytic evidence for abnormal neural response in dyslexic readers in OTC regions in deep and shallow orthographies. https://doi.org/10.1002/hbm.23202.

26. van der Mark S, Bucher K, Maurer U, Schulz E, Brem S, Buckelmüller J, et al. Children with dyslexia lack multiple specializations along the visual word-form (VWF) system. NeuroImage. 2009;47(4):1940-9. https://doi.org/10.1016/j.neuroimage.2009.05. 021.

27. Wimmer H, Schurz M, Sturm D, Richlan F, Klackl J, Kronbichler $\mathrm{M}$, et al. A dual-route perspective on poor reading in a regular orthography: an fMRI study. Cortex. 2010;46(10):1284-98. https://doi.org/10.1016/j.cortex.2010.06.004.

28. Richlan F, Sturm D, Schurz M, Kronbichler M, Ladurner G, Wimmer H. A common left occipito-temporal dysfunction in developmental dyslexia and acquired letter-by-letter reading? PLoS One. 2010;5(8):e12073. https://doi.org/10.1371/journal.pone. 0012073.

29. Olulade OA, Flowers DL, Napoliello EM, Eden GF. Dyslexic children lack word selectivity gradients in occipito-temporal and inferior frontal cortex. Neuroimage Clin. 2015;7:742-54. https://doi. org/10.1016/j.nicl.2015.02.013.

30. Shaywitz BA, Shaywitz SE, Blachman BA, Pugh KR, Fulbright RK, Skudlarski P, et al. Development of left occipitotemporal systems for skilled reading in children after a phonologically-based intervention. Biol Psychiatry. 2004;55(9):926-33. https://doi.org/ 10.1016/j.biopsych.2003.12.019.

31. Heim S, Pape-Neumann J, van Ermingen-Marbach M, Brinkhaus M, Grande M. Shared vs. specific brain activation changes in dyslexia after training of phonology, attention, or reading. Brain Struct Funct. 2015;220(4):2191-207. A study revealing consistent amelioration of OTC signal after intervention irrespective of training type. $h$ ttps://doi.org/10.1007/s00429-014-0784-y.

32. Maurer U, Bucher K, Brem S, Benz R, Kranz F, Schulz E, et al. Neurophysiology in preschool improves behavioral prediction of reading ability throughout primary school. Biol Psychiatry. 2009;66(4):341-8. https://doi.org/10.1016/j.biopsych.2009.02. 031.

33. Carroll JM, Solity J, Shapiro LR. Predicting dyslexia using prereading skills: the role of sensorimotor and cognitive abilities. 
J Child Psychol Psychiatry. 2016;57(6):750-8. https://doi.org/10. 1111/jcpp.12488.

34. Yamada Y, Stevens C, Dow M, Harn BA, Chard DJ, Neville HJ. Emergence of the neural network for reading in five-year-old beginning readers of different levels of pre-literacy abilities: an fMRI study. NeuroImage. 2011;57(3):704-13. https://doi.org/10.1016/j. neuroimage.2010.10.057.

35. Raschle NM, Zuk J, Gaab N. Functional characteristics of developmental dyslexia in left-hemispheric posterior brain regions predate reading onset. Proc Natl Acad Sci U S A. 2012;109(6):2156-61. https://doi.org/10.1073/pnas.1107721109.

36. Ozernov-Palchik O, Gaab N. Tackling the "dyslexia paradox": reading brain and behavior for early markers of developmental dyslexia. Wiley Interdiscip Rev Cogn Sci. 2016;7(2):156-76. Important information on the combination of neuroimaging measures and behavioral measures to accurately identify dyslexia risk. https://doi.org/10.1002/wcs.1383.

37. Tanaka H, Black JM, Hulme C, Stanley LM, Kesler SR, WhitfieldGabrieli S, et al. The brain basis of the phonological deficit in dyslexia is independent of IQ. Psychol Sci. 2011;22(11):1442-51. https://doi.org/10.1177/0956797611419521.

38. Cutting LE, Clements-Stephens A, Pugh KR, Burns S, Cao A, Pekar JJ, et al. Not all reading disabilities are dyslexia: distinct neurobiology of specific comprehension deficits. Brain Connect. 2013;3(2):199-211. https://doi.org/10.1089/brain.2012.0116.

39.• Perrachione TK, Del Tufo SN, Winter R, Murtagh J, Cyr A, Chang $\mathrm{P}$, et al. Dysfunction of rapid neural adaptation in dyslexia. Neuron. 2016;92(6):1383-97. Important study on how neuronal adaptation in dyslexia is not only disrupted for reading material but other stimulus types as well. https://doi.org/10.1016/j.neuron. 2016.11.020.

40. Boros M, Anton J-L, Pech-Georgel C, Grainger J, Szwed M, Ziegler JC. Orthographic processing deficits in developmental dyslexia: beyond the ventral visual stream. Neuroimage. 2016;128: 316-27. Recent study highlighting dyslexic impairments in early visual orthographic processing during symbol string processing. https://doi.org/10.1016/j.neuroimage.2016.01.014.

41. Monzalvo K, Fluss J, Billard C, Dehaene S, Dehaene-Lambertz G. Cortical networks for vision and language in dyslexic and normal children of variable socio-economic status. NeuroImage. 2012;61(1):258-74. https://doi.org/10.1016/j.neuroimage.2012.02. 035 .

42. Galaburda AM. Developmental dyslexia and animal studies: at the interface between cognition and neurology. Cognition. 1994;50(13):133-49. https://doi.org/10.1016/0010-0277(94)90025-6.

43. Kronbichler M, Wimmer H, Staffen W, Hutzler F, Mair A, Ladurner G. Developmental dyslexia: gray matter abnormalities in the occipitotemporal cortex. Hum Brain Mapp. 2008;29(5):613-25. https://doi.org/10.1002/hbm.20425.

44. Silani G, Frith U, Demonet J-F, Fazio F, Perani D, Price C, et al. Brain abnormalities underlying altered activation in dyslexia: a voxel based morphometry study. Brain. 2005;128(10):2453-61. https:// doi.org/10.1093/brain/awh579.

45. Richlan F, Kronbichler M, Wimmer H. Structural abnormalities in the dyslexic brain: a meta-analysis of voxel-based morphometry studies. Hum Brain Mapp. 2013;34(11):3055-65. https://doi.org/ 10.1002/hbm.22127.

46. Linkersdörfer J, Lonnemann J, Lindberg S, Hasselhorn M, Fiebach CJ. Grey matter alterations co-localize with functional abnormalities in developmental dyslexia: an ALE meta-analysis. PLoS One. 2012;7(8):e43122. https://doi.org/10.1371/journal.pone.0043122.

47. Altarelli I, Monzalvo K, Iannuzzi S, Fluss J, Billard C, Ramus F, et al. A functionally guided approach to the morphometry of occipitotemporal regions in developmental dyslexia: evidence for differential effects in boys and girls. J Neurosci. 2013;33(27): 11296-301. https://doi.org/10.1523/JNEUROSCI.5854-12.2013.
48. Williams VJ, Juranek J, Cirino P, Fletcher JM. Cortical thickness and local gyrification in children with developmental dyslexia. Cereb. Cortex. 2017; https://doi.org/10.1093/cercor/bhx001.

49. Bailey S, Hoeft F, Aboud K, Cutting L. Anomalous gray matter patterns in specific reading comprehension deficit are independent of dyslexia. Ann Dyslexia. 2016;66(3):256-74. https://doi.org/10. 1007/s11881-015-0114-y.

50. Im K, Raschle NM, Smith SA, Ellen Grant P, Gaab N. Atypical sulcal pattern in children with developmental dyslexia and at-risk kindergarteners. Cereb. Cortex. 2016;26(3):1138-48. An advanced morphometry study on structural abnormalities linked to dyslexia and risk for dyslexia. https://doi.org/10.1093/cercor/ bhu305.

51. Raschle NM, Chang M, Gaab N. Structural brain alterations associated with dyslexia predate reading onset. NeuroImage. 2011;57(3):742-9. https://doi.org/10.1016/j.neuroimage.2010.09. 055.

52. Raschle NM, Becker BLC, Smith S, Fehlbaum LV, Wang Y, Gaab $\mathrm{N}$. Investigating the influences of language delay and/or familial risk for dyslexia on brain structure in 5-year-olds. Cereb Cortex. 2017;27(1):764-76. https://doi.org/10.1093/cercor/bhv267.

53. Skeide MA, Kirsten H, Kraft I, Schaadt G, Müller B, Neef N, et al. Genetic dyslexia risk variant is related to neural connectivity patterns underlying phonological awareness in children. Neuroimage. 2015;118:414-21. An extremely important study on the left OTC structure combining showing influences of a genetic risk factor for dyslexia and including data from dyslexic children and children at-risk for dyslexia. https://doi.org/10.1016/j. neuroimage.2015.06.024.

54. Ulfarsson MO, Walters GB, Gustafsson O, Steinberg S, Silva A, Doyle OM, et al. $15 \mathrm{q} 11.2 \mathrm{CNV}$ affects cognitive, structural and functional correlates of dyslexia and dyscalculia. Transl Psychiatry. 2017;7(4):e1109. https://doi.org/10.1038/tp.2017.77.

55.• Eckert MA, Berninger VW, Vaden KI, Gebregziabher M, Tsu L. Gray matter features of reading disability: a combined metaanalytic and direct analysis approach $(1,2,3,4)$. eNeuro. 2016;3(1). An informative study from the important Dylsexia Data Consortium performing a large-scale analysis of gray matter in dyslexia. https://doi.org/10.1523/ENEURO.0103-15.2015.

56. Geschwind N. Disconnexion syndromes in animals and man. Brain. 1965;88(2):237-94. https://doi.org/10.1093/brain/88.2.237.

57. Vandermosten M, Boets B, Wouters J, Ghesquière P. A qualitative and quantitative review of diffusion tensor imaging studies in reading and dyslexia. Neurosci Biobehav Rev. 2012;36(6):1532-52. https://doi.org/10.1016/j.neubiorev.2012.04.002.

58. Vandermosten M, Vanderauwera J, Theys C, De Vos A, Vanvooren S, Sunaert S, et al. A DTI tractography study in pre-readers at risk for dyslexia. Dev Cogn Neurosci. 2015;14:8-15. This study suggests an important specific role of ventral white matter tracts in children at-risk for dyslexia before learning to read. https://doi. org/10.1016/j.den.2015.05.006.

59. Vanderauwera J, Wouters J, Vandermosten M, Ghesquière P. Early dynamics of white matter deficits in children developing dyslexia. Dev Cogn Neurosci. 2017;27:69-77. https://doi.org/10.1016/j.den. 2017.08.003.

60. Vandermosten M, Boets B, Poelmans H, Sunaert S, Wouters J, Ghesquière P. A tractography study in dyslexia: neuroanatomic correlates of orthographic, phonological and speech processing. Brain. 2012;135(3):935-48. https://doi.org/10.1093/brain/awr363.

61. Kraft I, Schreiber J, Cafiero R, Metere R, Schaadt G, Brauer J, et al. Predicting early signs of dyslexia at a preliterate age by combining behavioral assessment with structural MRI. NeuroImage. 2016;143:378-86. https://doi.org/10.1016/j.neuroimage.2016.09. 004.

62.• Schurz M, Wimmer H, Richlan F, Ludersdorfer P, Klackl J, Kronbichler M. Resting-state and task-based functional brain 
connectivity in developmental dyslexia. Cerebral Cortex. 2014;25: 3502-14. The first study to explore functional brain connectivity during task performance and rest in dyslexia.

63. Shaywitz SE, Shaywitz BA, Fulbright RK, Skudlarski P, Mencl WE, Constable RT, et al. Neural systems for compensation and persistence: young adult outcome of childhood reading disability. Biol Psychiatry. 2003;54(1):25-33. https://doi.org/10.1016/S00063223(02)01836-X.

64. van der Mark S, Klaver P, Bucher K, Maurer U, Schulz E, Brem S, et al. The left occipitotemporal system in reading: disruption of focal fMRI connectivity to left inferior frontal and inferior parietal language areas in children with dyslexia. NeuroImage. 2011;54(3): 2426-36. https://doi.org/10.1016/j.neuroimage.2010.10.002.

65. Zhou W, Xia Z, Bi Y, Shu H. Altered connectivity of the dorsal and ventral visual regions in dyslexic children: a resting-state fMRI study. Front Hum Neurosci. 2015;9:495.

66. Finn ES, Shen X, Holahan JM, Scheinost D, Lacadie C, Papademetris X, et al. Disruption of functional networks in dyslexia: a whole-brain, data-driven analysis of connectivity. Biol Psychiatry. 2014;76(5):397-404. https://doi.org/10.1016/j. biopsych.2013.08.031.

67. Stanberry LI, Richards TL, Berninger VW, Nandy RR, Aylward $\mathrm{EH}$, Maravilla KR, et al. Low-frequency signal changes reflect differences in functional connectivity between good readers and dyslexics during continuous phoneme mapping. Magn Reson Imaging. 2006;24(3):217-29. https://doi.org/10.1016/j.mri.2005. 12.006 .

68. Richards TL, Berninger VW. Abnormal fMRI connectivity in children with dyslexia during a phoneme task: before but not after treatment. J Neurolinguistics. 2008;21(4):294-304. https://doi.org/ 10.1016/j.jneuroling.2007.07.002.

69. Farris EA, Odegard TN, Miller HL, Ring J, Allen G, Black J. Functional connectivity between the left and right inferior frontal lobes in a small sample of children with and without reading difficulties. Neurocase. 2011;17(5):425-39. https://doi.org/10.1080/ 13554794.2010 .532141 .

70. Koyama MS, Di Martino A, Kelly C, Jutagir DR, Sunshine J, Schwartz SJ, et al. Cortical signatures of dyslexia and remediation: an intrinsic functional connectivity approach. PLoS One. 2013;8(2):e55454. https://doi.org/10.1371/journal.pone.0055454.

71. Richardson FM, Seghier ML, Leff AP, Thomas MSC, Price CJ. Multiple routes from occipital to temporal cortices during reading. J Neurosci. 2011;31(22):8239-47. https://doi.org/10.1523/ JNEUROSCI.6519-10.2011.

72. Cao F, Bitan T, Booth JR. Effective brain connectivity in children with reading difficulties during phonological processing. Brain Lang. 2008;107(2):91-101. https://doi.org/10.1016/j.bandl.2007. 12.009 .

73. Morken F, Helland T, Hugdahl K, Specht K. Reading in dyslexia across literacy development: a longitudinal study of effective connectivity. Neuroimage. 2017;144(Pt A):92-100. The first longitudinal DCM effective brain connectivity study on normal and impaired reading development. https://doi.org/10.1016/j. neuroimage.2016.09.060.
74. Saygin ZM, Osher DE, Norton ES, Youssoufian DA, Beach SD, Feather J, et al. Connectivity precedes function in the development of the visual word form area. Nat Neurosci. 2016;19(9):1250-5. https://doi.org/10.1038/nn.4354.

75. Stevens WD, Kravitz DJ, Peng CS, Tessler MH, Martin A. Privileged functional connectivity between the visual word form area and the language system. J Neurosci. 2017;37(21):5288-97. https://doi.org/10.1523/JNEUROSCI.0138-17.2017.

76. Dehaene $\mathrm{S}$, Cohen L. The unique role of the visual word form area in reading. Trends Cogn. Sci. (Regul. Ed.). 2011;15(6):254-62. https://doi.org/10.1016/j.tics.2011.04.003.

77. Dehaene S, Le Clec'H G, Poline J-B, Le Bihan D, Cohen L. The visual word form area: a prelexical representation of visual words in the fusiform gyrus. Neuroreport. 2002;13(3):321-5. https://doi.org/ 10.1097/00001756-200203040-00015.

78. Glezer LS, Jiang X, Riesenhuber M. Evidence for highly selective neuronal tuning to whole words in the "visual word form area.". Neuron. 2009;62(2):199-204. https://doi.org/10.1016/j.neuron. 2009.03.017.

79. Kronbichler M, Bergmann J, Hutzler F, Staffen W, Mair A, Ladurner G, et al. Taxi vs. taksi: on orthographic word recognition in the left ventral occipitotemporal cortex. J Cogn Neurosci. 2007;19(10):1584-94. https://doi.org/10.1162/jocn.2007.19.10. 1584.

80. Ludersdorfer P, Kronbichler M, Wimmer H. Accessing orthographic representations from speech: the role of left ventral occipitotemporal cortex in spelling. Hum Brain Mapp. 2015;36(4):1393-406. https://doi.org/10.1002/hbm.22709.

81. Price CJ, Devlin JT. The interactive account of ventral occipitotemporal contributions to reading. Trends Cogn Sci. 2011;15(6):246-53. https://doi.org/10.1016/j.tics.2011.04.001.

82. Sebastian R, Gomez Y, Leigh R, Davis C, Newhart M, Hillis AE. The roles of occipitotemporal cortex in reading, spelling, and naming. Cogn Neuropsychol. 2014;31(5-6):511-28. https://doi.org/10. 1080/02643294.2014.884060.

83. Cohen L, Jobert A, Le Bihan D, Dehaene S. Distinct unimodal and multimodal regions for word processing in the left temporal cortex. NeuroImage. 2004;23(4):1256-70. https://doi.org/10.1016/j. neuroimage.2004.07.052.

84. Brodersen KH, Deserno L, Schlagenhauf F, Lin Z, Penny WD, Buhmann JM, et al. Dissecting psychiatric spectrum disorders by generative embedding. NeuroImage: Clinical. 2014;4:98-111. https://doi.org/10.1016/j.nicl.2013.11.002.

85. Weiskopf N, Mohammadi S, Lutti A, Callaghan MF. Advances in MRI-based computational neuroanatomy: from morphometry to invivo histology. Curr Opin Neurol. 2015;28(4):313-22. https://doi. org/10.1097/WCO.0000000000000222.

86. Di Martino A, Yan C-G, Li Q, Denio E, Castellanos FX, Alaerts K, et al. The autism brain imaging data exchange: towards a large-scale evaluation of the intrinsic brain architecture in autism. Mol Psychiatry. 2014;19(6):659-67. https://doi.org/10.1038/mp.2013. 78. 